\title{
A cytological comparison of efficacy of bronchioalveolar lavage, transbronchial needle aspiration and image guided fine needle aspiration for diagnosis of pulmonary lesions in a rural tertiary care centre
}

\author{
Ivreet Kaur ${ }^{1}$, Eshita Dadwal ${ }^{2}$, Sanjay Bedi ${ }^{3}$,", Sameer Singhal ${ }^{4}$, Amit Mittal $^{5}$ \\ ${ }^{1}$ Assistant Professor, ${ }^{2}$ Junior Resident, ${ }^{3}$ Professor, Dept. of Pathology, ${ }^{4}$ Professor, Dept. of Pulmonary Medicine, \\ ${ }^{5}$ Professor, Dept. of Radiodiagnosis, MMIMSR, Mullana-Ambala, Haryana
}

*Corresponding Author:

Email: drsanjaybedi@gmail.com

\begin{abstract}
Introduction: Pulmonary lesions include a wide variety of benign and malignant conditions of lung, pleura, mediastinum and vertebrae. Lung cancer is a frequently diagnosed cancer worldwide. ${ }^{1}$ Although clinical data, location and radiological findings can narrow down the diagnostic possibilities, a cytological diagnosis is warranted before initiating the specific treatment for the malignant disease.

Aims and Objectives: The present study was undertaken with the aim to compare the sensitivity and specificity of bronchioalveolar lavage (BAL), transbronchial needle aspiration cytology (TBNA) and image-guided fine needle aspiration cytology (FNAC) in the evaluation of patients with pulmonary lesions

Materials and Methods: The present study was conducted on 100 patients with pulmonary lesions coming to the department of pulmonary medicine at MMIMSR, Mullana. For diagnostic purposes various procedures like BAL, TBNA and image guided FNAC were performed. Cytological examination was carried out in the department of Pathology.

Results: Among 100 cases, TBNA was done in 63 cases, BAL in 52 cases and image guided FNAC was performed in 37 cases. Using these cytological techniques, a diagnosis was obtained in $81 \%$ cases. The sensitivity and specificity of each technique was assesed. On cytological evaluation, $12 \%$ cases had a non-neoplastic diagnosis, whereas $69 \%$ cases, malignancy was diagnosed. However, in $19 \%$ cases no diagnosis was offered. The most common diagnosis amongst malignant lesions was non-small cell carcinomas. (Squamous cell carcinoma- 28\%, Adenocarcinoma-23\%) followed by small cell carcinoma (8\%).

Conclusion: Image-guided FNAC of pulmonary lesions is a simple, safe, economically viable technique with low morbidity and offers quick and early diagnosis. Our study revealed that TBNA and FNAC were comparatively better in the diagnosis when compared to BAL.
\end{abstract}

Keywords: Image-guided Fine Needle Aspiration Cytology, Pulmonary Lesions, Squamous Cell Carcinoma.

Received: $13^{\text {th }}$ July, 2017

\section{Introduction}

Lung cancer is a frequently diagnosed cancer in the world and a common cause of death related to cancer. Lung cancer comprises $17 \%$ of the total newly diagnosed cancer cases in males and causes $23 \%$ of total cancer related deaths. ${ }^{2}$ The prognosis of lung cancer depends on the stage of the cancer at the initial diagnosis. Therefore, an early diagnosis of lung cancer improves the prognosis of lung cancer. ${ }^{3}$ Different modalities used for early diagnosis are radiological investigations, TBNA, bronchial biopsy, exfoliative cytology like bronchial washings, BAL and image guided FNAC. ${ }^{4}$

Image-guided FNAC is well established diagnostic tool for diagnosis of radiological apparent lesions of the lung. The procedure has a low complication rate and is highly sensitive for the diagnosis of non-palpable deep-seated lung lesions. ${ }^{5-9}$ TBNA of the mediastinum is rapidly
Accepted: $07^{\text {th }}$ October, 2017

becoming a technique of choice for minimally invasive staging of lung cancer. ${ }^{10}$

BAL via a flexible bronchoscope was introduced by Reynolds and Newball in 1974 as a therapeutic procedure for clearing secretions in the alveolar spaces. ${ }^{11,12}$ Subsequently, the technique has been used as diagnostic procedure for confirmation of pulmonary infections particularly in immunocompromised individuals and interstitial lung diseases, thereby replacing the need of open biopsy. ${ }^{13}$

These cytological procedures are easily performed and well tolerated for routine assessment of the patients with lung lesions. While correlating these cytological techniques false negative and false positive results can be reduced, thereby, improving the diagnostic efficacy. These cytological procedures have many advantages but more work requires to be done. The present study is undertaken with the aim to study the efficacy of BAL, TBNA and image 
guided fine needle aspiration in the evaluation of patients with pulmonary lesions.

\section{Materials and Method}

The present study was conducted on 100 patients with pulmonary lesions coming to the department of respiratory medicine, MMIMSR at Mullana over the time period June 2014 to December 2016. Ethical clearance for the study was obtained from institute ethical committee. Informed consent was obtained from all the patients after briefly explaining the technique. The patient's requisition forms including patient's age, sex, smoking history and radiological investigations were filled by clinicians. For diagnostic purposes, procedures like BAL and TBNA were performed in the department of pulmonary medicine. Image-guided FNAC were done in the department of radio-diagnosis, with the help of lumbar puncture needle No. 20-22, attached to $20 \mathrm{ml}$ disposable syringe with Franzen handle through percutaneous and transthoracic approach. The collected material was smeared on the slides. Half of slides were wet fixed whereas the rest of the slides were air-dried and sent to the department of pathology. Wet fixed slides were stained with hematoxylin \& eosin stain and papanicolaou (PAP) stains, while air-dried smears were stained with Leishman-Giemsa. Final diagnosis was made with the help of bronchoscopic findings, clinico-radiologic impressions, cytological findings and response to treatment.

\section{Observations and Results}

Hundred cases were included in the present study. Among these 100 cases, both BAL and TBNA were performed in 52 cases whereas in 11 cases TBNA alone was done. Among 37 cases of image-guided FNAC, 33 cases were ultrasoundguided and 4 cases were computed tomograghy (CT) guided (Table1). Age of the patients was between 14 years to 80 years (mean 53.14 years). Male female ratio was 2.7:1 having a male prepondrance. Majority of patients had history of smoking. In our study, the most common presenting complaint was cough (73\%) followed by shortness of breath $(63 \%)$ and chest pain (53\%). The lung lesions were central in 63\% and the peripheral in $37 \%$ cases.

Of the 100 cases, a firm diagnosis was offered in 81 cases using various cytological techniques. In 62 patients where TBNA was performed, diagnosis was possible in 46 cases whereas in 17 cases no diagnosis could be arrived at. The sensitivity and specificity of TBNA was
$86.3 \%$ and $83.3 \%$. BAL was done in 52 cases and correct diagnosis was achieved in 14 cases. The sensitivity and specificity of BAL procedure in our study was $34.2 \%$ and $90.9 \%$, respectively. In cases of image-guided FNAC, the correct diagnosis was obtained in 35 cases. Thus, the sensitivity and specificity of image guided procedure was $97.2 \%$ and $100 \%$ respectively.

The lesions were catagorized as nonneoplastic, neoplastic and non-diagnostic. In nonneoplastic category, there were 6 cases each of granulomatous inflammation (Fig. 1A and 1B) and non-specific inflammatory pathology. Of the six cases of granulomatous inflammation, two cases were Ziehl-Neelson positive and thus, these were labelled as tuberculosis. In the neoplastic category, no benign lesion was encountered in the study. Study showed 69 malignant cases when 8 cases were diagnosed as small cell carcinoma, 28 cases squamous cell carcinoma, 23 cases adenocarcinoma and 4 cases poorly differentiated carcinoma (Table 2).

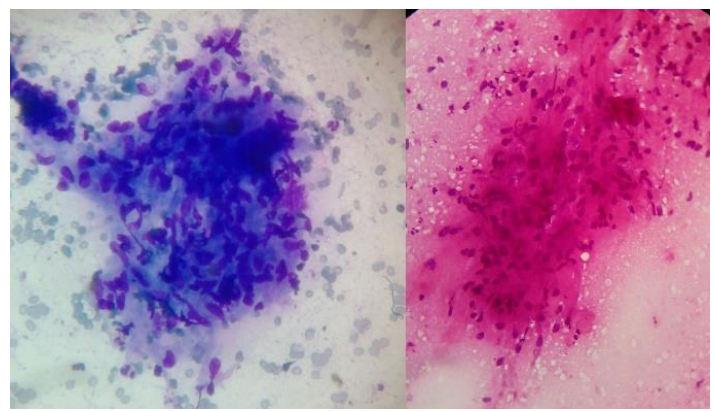

Fig. 1a, b: a- Photomicrograph of cytological smear showing epithelioid cell granuloma. (Leishman-Giemsa 400X), b- Smear showing epithelioid cell granuloma with lymphoid cells. (Hematoxylin \& Eosin $\mathbf{4 0 0 X}$ )

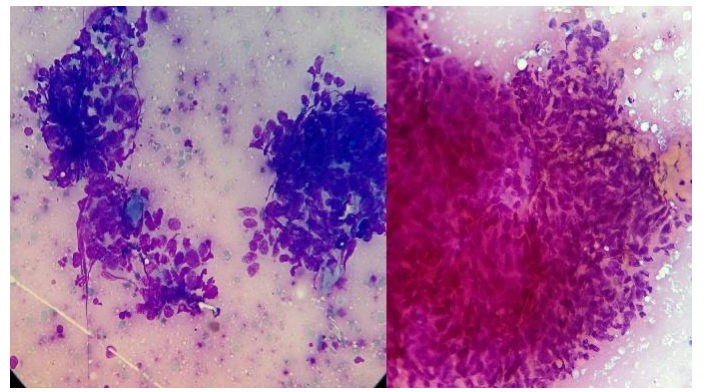

Fig. 2a, b: a- Photomicrograph revealing clusters of atypical squamous cells in background of keratinous debris. (LeishmanGiemsa 400X), b- Smear showing clusters of tumor cells exhibiting spindling in case of squamous cell carcinoma. (Pap stain 400X) 


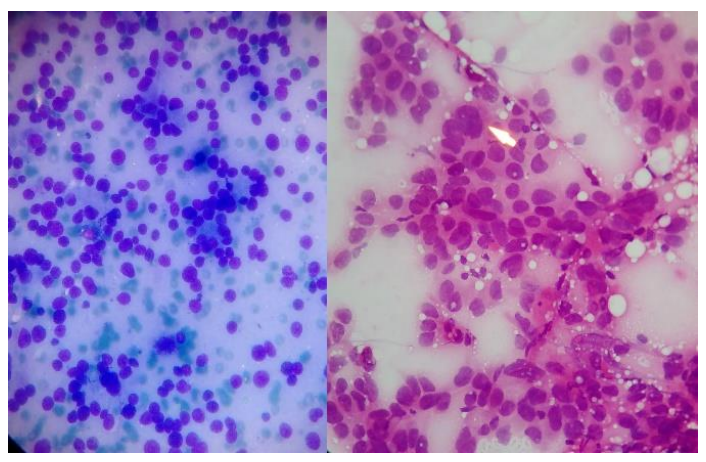

Fig. 3a, b: a- Photomicrograph of adenocarcinoma exhibiting gland formation.(Leishman-Giemsa 400X), bSmear showing dispersed tumor cells of adenocarcinoma exhibiting nuclear pleomorphism with vague gland formation. (Hematoxylin \& Eosin 400X)

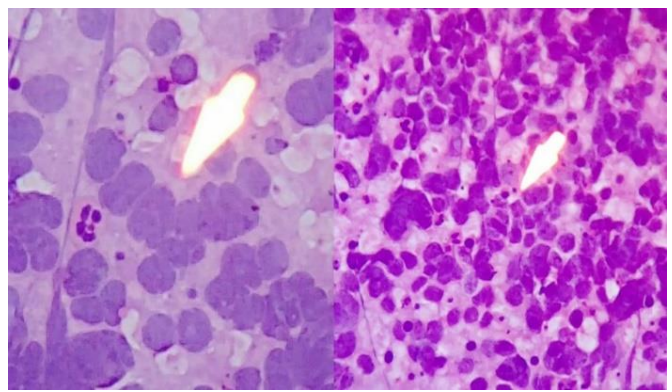

Fig. 4a, b: a- Small cell carcinoma- tumor cells revealing nuclear moulding and nuclear streaking. (Leishman-Giemsa 400X), bPhotomicrograph revealing tumor cells with hyperchromatic nucleus exhibiting nuclear moulding. (Hematoxylin \& Eosin 400X)

Table 1: Distribution of cases according to the procedure performed

\begin{tabular}{|l|c|}
\hline \multicolumn{1}{|c|}{ Type of procedure } & No. of cases \\
\hline TBNA and BAL & 52 \\
\hline TBNA & 11 \\
\hline Image guided FNAC & 37 \\
\hline Total & 100 \\
\hline
\end{tabular}

Table 2: Cytological diagnosis of 100 cases of Pulmonary lesions

\begin{tabular}{|l|c|}
\hline \multicolumn{1}{|c|}{ Cytologic diagnosis } & $\begin{array}{c}\text { No. of } \\
\text { cases }\end{array}$ \\
\hline Squamous cell carcinoma & 28 \\
\hline Adenocarcinoma & 23 \\
\hline Small cell carcinoma & 08 \\
\hline Granulomatous inflammation & 06 \\
\hline $\begin{array}{l}\text { Non- specific inflammatory } \\
\text { pathology }\end{array}$ & 06 \\
\hline Poorly differentiated carcinoma & 04 \\
\hline Adenosquamous carcinoma & 01 \\
\hline Large cell carcinoma & 01 \\
\hline
\end{tabular}

\begin{tabular}{|l|c|}
\hline Adenoid cystic carcinoma & 01 \\
\hline $\begin{array}{l}\text { Hodgkins disease with } \\
\text { pulmonary involvement }\end{array}$ & 01 \\
\hline Carcinoid tumor & 01 \\
\hline Anaplastic large cell lymphoma & 01 \\
\hline Non-diagnostic & 19 \\
\hline Total & 100 \\
\hline
\end{tabular}

\section{Discussion}

The cytological evaluation in diagnosis of pulmonary lesions has been considered as one of its best application. The present study is one of a kind, as here, an attempt has been made to compare the various techniques in efficiently diagnosing various lesions of the lung.

The present study was done on 100 cases of pulmonary lesions using various cytological techniques. The mean age of the patients under study was 53.14 years which is comparable to studies done by Sareen et $\mathrm{al}_{1}{ }^{3}$ Bhat et $\mathrm{al}^{4}$ and Rohtagi et al. ${ }^{12}$ There was male preponderance of pulmonary lesions as mentioned by majority of the studies of the literature. The male: female ratio of present study was $2.7: 1$. Bodh et $\mathrm{al}^{14}$ found in their study that male: female ratio is $3.35: 1$ whereas Sareen et $\mathrm{al}^{3}$ showed male: female ratio of 8.4:1. Cough and shortness of breath was the most common complaints. Similar findings were observed in the study by Sareen et al. ${ }^{3}$

Three cytological techniques i.e. TBNA, BAL and image guided FNAC were performed for the evaluation of the lesions. Both TBNA and BAL were performed in 52 cases of radiologically suspected lung lesions. The sensitivity and specificity of BAL procedure was $34.2 \%$ and $90.9 \%$, respectively. The sensitivity of our study correlated however, the specificity was slightly higher than the study done by Bhat et al. ${ }^{4}$ In present study, 14 cases were correctly diagnosed, one case was false positive and twenty seven cases were false negative. The main reason for false negative cases was due to non-representative material or paucicellular smears. The cytological sampling by BAL procedure depends on exfoliated cells from the lesion. Further, it is dependent on technical skill of physician who is performing the lavage, differentiation of malignant growth, as poorly differentiated lesions are less cohesive and cytological preservation of material is obtained. While the exfoliative cells lying in bronchial cavity undergo degenerative changes and loose their morphological details making them difficult to differentiate from reactive bronchial epithelial cells. False positive cases can result from misinterpretation of cellular changes in inflammatory conditions such as pneumonia of long duration (atypical 
macrophages) and squamous metaplasia. In the present study, there was one false positive case due to misinterpretation of squamous metaplasia as suspicious of malignancy. As there were fewer number of false positive cases, this was the strength of BAL cytology in the present study.

TBNA was performed in 63 cases and the correct diagnosis was achieved in 44 cases. There were two false positive cases due to misinterpretation of squamous metaplasia as dysplastic squamous cells suggestive of malignancy. In a study conducted by $\mathrm{Ye} \mathrm{T}$ et al, ${ }^{10}$ the sensitivity and specificity of TBNA was $95.08 \%$ and $100 \%$, respectively which was slightly higher than our study. The relatively lower sensitivity and specificity of TBNA in our study was due to paucicellular smears which could be due to low technical expertise of the pulmonologist to obtain adequate representative sample from the lesion.

Current diagnosis was possible in 35 of the 37 cases of image guided FNAC. No false positive case was reported on the cytology, therefore, giving specificity as close to $100 \%$ and the sensitivity of $97.2 \%$. In a study done by Sareen et $\mathrm{al}^{3}{ }^{3}$ the sensitivity and specificity of CT guided FNAC were found to be $87.25 \%$ and $100 \%$, respectively which correlated well with our study. No complication was noted during or after the procedure in any of the cases. There was one false negative case in present study which on repeated aspirations yielded necrotic material only. The diagnosis was later confirmed on biopsy as squamous cell carcinoma.

Squamous cell carcinoma (Fig. 2A and 2B; $28 \%$ cases) was the most common malignancy followed by adenocarcinoma (Fig. 3A and 3B; $23 \%$ cases) and small cell carcinoma (Fig. $4 \mathrm{~A}$ and 4B; $8 \%$ cases). In western world, adenocarcinoma is the most common malignancy of the lung. ${ }^{15}$ However, most of the Indian studies mentions squamous cell carcinoma to be the most frequently occurring lung malignancy. In the present study, four cases were diagnosed as poorly differentiated carcinoma without furthur subtyping due to the presence few viable anaplastic cells in a necrotic background. The other less common malignancies diagnosed in our study were adenosquamous carcinoma (1case), large cell carcinoma (1 case), adenoid cystic carcinoma (1 case), carcinoid (1 case), Hodgkin's disease with pulmonary involvement (1 case) and anaplastic large cell lymphoma (1 case).

Thus, for pursuing diagnosis of pulmonary lesions especially lung cancer, there are number of cytological procedure to choose from. For central lesions, TBNA is the preferred technique whereas the peripheral lesions are best approached with image-guided FNAC. However, BAL is the safest procedure and by increasing the number of attempts while obtaining sample can increase its sensitivity and specificity especially in peripheral lung lesions.

\section{Conclusion}

Image-guided FNAC of pulmonary lesion is a simple, safe, economically prudent technique with low morbidity and leading to quick and early diagnosis. It was found in our study that TBNA and FNAC are comparatively more efficient than BAL even though BAL and TBNA can be done in one sitting.

\section{References}

1. Brambilla E, Travis WD, Colby TV, Corrin B, Shimosato Y. The new world health organization classification of lung tumors. Eur Respir J.2001;18:1059-68.

2. The Health Consequences of smoking: A report of surgeon General. U.S. department of health and human services, Public Health Service, Centers for Disease Control and Prevention, Washington, DC 2004, CDC Publication No.789.

3. Sareen R, Pandey CL. Lung malignancy: Diagnostic accuracies of bronchoalveolar lavage, bronchial brushing and fine needle aspiration cytology. Lung India 2016;33:635-41.

4. Bhat N, Nazeir MJ, Bashir N, Farooq S, Fatima K, Baba KM. Correlation of bronchial biopsy with bronchoalveolar lavage in lung malignancies. Int J Res Med Sci 2016;4(2):42835.

5. Redddy SA, Vivekanand N, Durga K. Evaluation of ultrasound guided fine needle aspiration cytology for diagnosing lung lesions- a 3 year study. Indian Journal of Mednodent 2015;3(1):15 .

6. Singh R, Mallik M, Rai HK, Singh S, Mallick S, Kumar M. CT guided FNAC of lung mass- A retrospective study of disease spectrum. Int J Med Res Rev 2016:4(7):1088-91.

7. Liang L, Liu J, Buryanek J, Zhang S. CT-guided core needle biopsy of peripheral lung lesions with onsite evaluation: review of 215 cases. J Cytol Histol 2014;S4:018.

8. Das S, Dasgupta S, Das AK, Bhattacharyya S, Ghosh I. Lung SOL- Cytology and histological correlation in a tertiary care centre. Journal of Dental and Medical Sciences 2015;4(6):18-24.

9. Madan M, Bannur H. Evaluation of fine needle aspiration cytology in diagnosis of lung lesions 2010;26(1):1-7.

10. Ye T, Hu H, Luo X, Chen H. The role of endobronchial ultrasound guided transbronchial needle aspiration (EBUS-TBNA) fot qualitative diagnosis of mediastinal and hilar lymphadenopathy: a prospective analysis. BMC Cancer 2011;11:100.

11. Reynolds HY, Newball HH. Analysis of proteins and respiratory cells obtained from human lungs 
by bronchial lavage. J Lab Clin Med

1974;84:559-73.

12. Rohtagi S, Khan L, Pantola C, Kumar A, Singh

PK. Correlation of fine needle aspiration with

bronchoalveolar lavage and brush smear cytology

in pulmonary lesions. Journal of Evolution of

Medicine and Dental Sciences2014;25(3):6957-

64.

13. Joos L, Chhajed PN, Wallner J, Battegay M,

Steiger J, Gratwohl A, Tamm M. Pulmonary infections diagnosed by BAL: A 12 year

experience in 1066 immunocompromised patients. Respiratory Medicine 2007;101:93-7.

14. Bodh A, Kausal V, Kashyap S, Gulati A.

Cytohistological correlation in diagnosis of lung tumors by using fibreoptic bronchoscopy: Study of 200 cases. Indian J Pathol Microbiol 2013;56(2):84-8

15. American Cancer Society. Statistics for 2006 . Available from: http://www.cancer.org/docrot/stt/stt_0.asp[Last assessed on 2015 Sep 23]. 